\title{
Monophosphoryl Lipid A as an adjuvant for immune therapy? A detailed in vitro comparison to LPS
}

\author{
Stefan Schülke ${ }^{1 *}$, Lothar Vogel ${ }^{2}$, Adam Flaczyk², Sonja Wolfheimer², Stefan Vieths², Stefan Scheurer ${ }^{2}$ \\ From 5th International Symposium on Molecular Allergology (ISMA 2013) \\ Vienna, Austria. 6-7 December 2013
}

Monophosphoryl lipid A (MPL) is a non-toxic TLR4 ligand, derived from Salmonella minnesota R595 (Re) lipopolysaccharide (LPS) by chemical modification. It is clinically used as an adjuvant for cancer treatment (Fendrix $^{\circledR}$, Ceravix $\left.^{\circledR}\right)$ and allergen specific immunotherapy (Pollinex ${ }^{\circledR}$ Quattro, ORALVAC ${ }^{\circledR}$ ). Nevertheless, reports on the mechanism of adjuvant activity are limited. The aim of this study was to compare the immune modulating capacities of MPL and LPS in vitro.

In both human and murine lung epithelial cell lines (LA-4, A549) LPS induced a higher CCL2 secretion than MPL. In murine BM-derived myeloid dendritic cells $(\mathrm{mDC})$, LPS as well as MPL stimulation resulted in the same pattern of cytokine secretion (IL-1 $1 \beta$, IL-6, IL-10 and TNF- $\alpha$ ). At high concentrations of MPL, IL- $1 \beta$ secretion was 4-fold higher compared to LPS, whereas LPS stimulation resulted in higher secretion of IL-6, IL-10 and TNF$\alpha$, respectively. Moreover, $\mathrm{mDC}$ stimulation with both adjuvants resulted in a pronounced cell activation pattern characterized by CD40 and CD69 upregulation, at which LPS proved to be more potent than MPL (thresholds for mDC activation: MPL: $100 \mathrm{ng} / \mathrm{ml}$, LPS: $1 \mathrm{ng} / \mathrm{ml}$ ). In ${\mathrm{MyD} 88^{-/-} \text {and Trif }}^{-{ }_{-}} \mathrm{mDC}$, MPL-induced cytokine secretion was absent in MyD88- but only reduced in Trif-deficient $\mathrm{mDC}$. LPS induced cytokine secretion was mostly unchanged in Trif ${ }^{1-} \mathrm{mDC}$. Furthermore, the co-administration of MPL and Ova resulted in enhanced IFN- $\gamma$ and IL-5 secretion from OVA-specific DO11.10 $\mathrm{CD}^{+}{ }^{+} \mathrm{T}$ cells co-cultured with BALB/c mDC which was not observed for LPS controls. In line with this result, stimulation with a covalent fusion protein of MPL and Ova (MPL:Ova) resulted in enhanced cytokine secretion from both $\mathrm{mDC}$
(IL-1 $\beta$, IL-6, TNF, IL-10, IL-12) and CD4 T cells (IL-5, IL-13, IL-2, IFN- $\gamma$, IL-17) compared to equimolar concentrations of MPL and Ova provided individually or as a mixture. Interestingly, Ova induced IL-9 secretion from $\mathrm{CD} 4^{+} \mathrm{T}$ cells was dose-dependently repressed when fused to MPL.

In summary, using in vitro assay systems we observed similar but attenuated immune responses induced by MPL in comparison to LPS. MPL applied together with allergen (either mixed or covalently fused) on $\mathrm{CD} 4^{+}$ $\mathrm{T}$ cells boosted allergen-specific TH1-, TH2-, and TH17adaptive responses. Although considered safe in humans, further studies should critically assess the adjuvant capacity of MPL in order to evaluate potential non-desired immunological effects.

\section{Authors' details}

'Paul-Ehrlich-Institut, Paul-Ehrlich Straße 51-59, Langen (Hessen), 63225, Germany. ${ }^{2}$ Paul-Ehrlich Institut, Division of Allergology, Langen, Germany.

Published: 17 March 2014

doi:10.1186/2045-7022-4-S2-O21

Cite this article as: Schülke et al: Monophosphoryl Lipid A as an adjuvant for immune therapy? A detailed in vitro comparison to LPS. Clinical and Translational Allergy 2014 4(Suppl 2):O21. 